\title{
Toward the Generation of a Wind Geophysical Model Function for Spaceborne GNSS-R
}

\author{
Wenming Lin ${ }^{\circledR}$, Senior Member, IEEE, Marcos Portabella ${ }^{\circledR}$, Giuseppe Foti ${ }^{\circledR}$, \\ Ad Stoffelen, Senior Member, IEEE, Christine Gommenginger, \\ and Yijun $\mathrm{He}^{\mathbb{1}}$, Member, IEEE
}

\begin{abstract}
This paper presents a comprehensive procedure to improve the wind geophysical model function (GMF) for the Global Navigation Satellite System Reflectometry (GNSS-R) instrument onboard the TechDemoSat-1 satellite. The observable used to define the GMF is extracted from the measured delayDoppler maps (DDMs) by correcting for the nongeophysical effects within the measurements. Besides the instrument and the geometric effects as provided in the bistatic radar equation, a calibration term that accounts for the uncalibrated receiver antenna gain and the unknown transmitter antenna gain is proposed to optimize the calculation of GNSS-R observables. Such calibration term is presented as a function of observing elevation and azimuth angles and is shown to remarkably reduce the measurement uncertainties. First, an empirical wind-only GMF is developed using the collocated Advanced Scatterometer (ASCAT) winds and European Centre for Medium-Range Weather Forecasts (ECMWF) model wind output. This empirical GMF agrees well with the model output. Then, the sensitivity of the observable to waves is analyzed using the collocated ECMWF wave parameters. The results show that it is difficult to include mean square slope (MSS) in the development of an empirical GMF, since the difference between ECMWF MSS and the MSS sensed by GNSS-R varies with incidence angle and wind speed. However, it is relevant to take significant wave height $\left(H_{S}\right)$ in account, particularly for low wind conditions. Consequently, a wind $/ H_{S}$ approach is proposed for improved wind retrievals.
\end{abstract}

Index Terms-Advanced Scatterometer (ASCAT), calibration, Global Navigation Satellite System Reflectometry (GNSS-R), wave, winds.

Manuscript received January 26, 2018; revised May 21, 2018 and June 22, 2018; accepted July 15, 2018. This work was supported in part by the National Natural Science Foundation of China under Grant 41706197, in part by the Startup Foundation for Introducing Talent of NUIST, and in part by the European Space Agency Project under Reference 400011699/16/NL/CT. (Corresponding author: Wenming Lin.)

W. Lin was with the Institute of Marine Sciences, 08003 Barcelona, Spain. He is now with the School of Marine Sciences, Nanjing University of Information Science and Technology, Nanjing 210044, China (e-mail: wenminglin@nuist.edu.cn).

M. Portabella is with the Institute of Marine Sciences, 08003 Barcelona, Spain (e-mail: portabella@icm.csic.es).

G. Foti and C. Gommenginger are with the National Oceanography Centre, Southampton SO14 3ZH, U.K. (e-mail: g.foti@noc.ac.uk; cg1@noc.ac.uk).

A. Stoffelen is with the Royal Netherlands Meteorological Institute, 3730 AE De Bilt, The Netherlands (e-mail: ad.stoffelen@knmi.nl).

$\mathrm{Y}$. $\mathrm{He}$ is with the School of Marine Sciences, Nanjing University of Information Science and Technology, Nanjing 210044, China (e-mail: yjhe@nuist.edu.cn).

Color versions of one or more of the figures in this paper are available online at http://ieeexplore.iee.org.

Digital Object Identifier 10.1109/TGRS.2018.2859191

\section{INTRODUCTION}

$\mathbf{G}$ LOBAL Navigation Satellite System Reflectometry (GNSS-R) techniques have shown their capability to observe many geophysical parameters, such as soil moisture [1]-[3], snow and sea ice characterization [4]-[6], and sea surface wind speed [7]-[9]. Similar to the operational scatterometer level 2 (L2) processing, the GNSS-R wind inversion relies on a geophysical model function (GMF) which, for the latter, relates the measured delay-Doppler map (DDM) to the sea surface wind speed for a certain observing geometry. For instance, the National Oceanographic Centre has developed different wind inversion algorithms for the Space GNSS receiver-remote sensing instrument (SGR-ReSI) onboard the U.K. TechDemoSat-1 (TDS-1) satellite [10], [11]. Both algorithms are based on the signal-to-noise (SNR) ratio defined as the ratio of the averaged signal value from a box surrounding the peak of DDM and the averaged noise value from a box within the signal-free area of the DDM. The fastdelivery inversion (FDI) algorithm in [10] is implemented in the Measurement of Earth Reflected Radio-Navigation Signals By Satellite (MERRByS) ground processing system to produce and distribute L2 wind speed products to potential end-users. It simply relates the sea surface wind speed to SNR and receiver antenna gain by a power law. A more sophisticated algorithm based on the bistatic radar equation (BRE) is proposed in [11] to correct not only for the receiver antenna gain but also for the GNSS-R bistatic viewing geometry, in order to derive an observable that better represents surface winds, and in turn, to improve the retrieved wind quality. It also uses a power-law empirical relationship between the derived observable and the reference Advanced Scatterometer (ASCAT) wind speed. Although BRE leads to higher retrieved wind quality than FDI, it is much less efficient than the latter in terms of ground processing, thus not suitable for a rapid data delivery [10]. Similar empirical approaches are developed to retrieve wind speed from the Cyclone GNSS (CYGNSS) mission [12]-[14]. In [12] the wind speeds are estimated from five different observables derived from GNSS$\mathrm{R}$ DDMs, based on empirical regressions of the observables against buoy wind. Then a minimum variance estimator is presented to composite the five wind estimates into a unique wind speed value with lower error than that of each individual wind estimate. This approach is improved by several additional 
processing steps in [13], such as by decoupling the effects of the geometry and the Woodward ambiguity function, and by optimizing the composition of different wind estimates. More recently, a Bayesian wind speed estimator is developed to correct for the long-wave sensitivity at low wind speeds [14]. Basically, this approach requires a characterization of the observable as a function of wind speed and significant wave height. In summary, regardless of the inversion algorithm used, one needs to define one (several) observable(s) from the measured DDMs, regress them against the collocated reference winds and/or other geophysical parameters, quantify the relationship between the observable(s) and such parameters through an empirical lookup table (LUT), and incorporate the LUT into a retrieval algorithm.

In this paper, we aim to develop an improved wind GMF for SGR-ReSI based on the level 1B (L1B) data disseminated by MERRByS. The fundamental idea consists of two parts. First, the improved GMF should include a GNSS-R observable which correlates well with the surface winds. Consequently, an approach similar to the BRE algorithm is applied to remove the nongeophysical effects from the GNSS-R DDMs, such as the observing geometry, the antenna gain pattern, and the instrumental calibration factors. Second, the GNSS-R signal results from both the specular reflection and the diffuse scattering of microwaves off the sea surface. The coherent reflection part is expected to be dominated by large waves, while the diffuse scattering part mainly depends on short waves. According to the current GNSS-R theory [7], the DDM in theory is fully characterized by the mean-square slope (MSS), which depends on the local wind speed, the fetch, and the incoming swell. If accurate wave reference data were widely available and if GNSS-R indeed responded to large waves, then an MSS GMF may be more suitable than a wind GMF, since it accounts for the swell effects. On the other hand, accurate sea surface wind measurements are widely available, which favors the development of a wind GMF. Note though that sea-state-dependent effects, other than those produced by the local wind, i.e., swell, should be taken into account in the GMF development. Such effects can, for instance, be modeled by analyzing significant wave height $\left(H_{S}\right)$ dependencies in low wind conditions. Therefore, although the observable is well described by the sea surface wind speed signature, other effects, such as sea surface temperature (SST) and significant wave height $\left(H_{S}\right)$, are also taken into account during the GMF development as second-order improvements [15].

Section II introduces the ancillary data and the simulator used in this paper. Then a set of empirical criteria are presented to select only the SGR-ReSI data of relatively high quality. In Section III, the analysis of both simulated and real TDS-1 is carried out in order to develop the corrections for removing the mentioned nongeophysical effects. In Section IV, the relevant corrections that are verified in the simulations are applied to the real TDS-1 data, and an empirical wind-only GMF is generated. Then, the sensitivity of TDS- 1 corrected observations to sea surface conditions is analyzed using collocated ASCAT level 2 (L2) wind data and the European Centre for Medium-Range Weather Forecasts (ECMWF) model output, e.g., winds and waves. A combined wind $/ H_{S} \mathrm{GMF}$ is proposed
TABLE I

List OF THE COLLOCATED ANCILLARy PARAMETERS

\begin{tabular}{|l|l|}
\hline Parameters & Source \\
\hline Wind speed and direction & ECMWF/ASCAT \\
\hline Significant wave height $\left(H_{\mathrm{s}}\right)$ & ECMWF \\
\hline $\begin{array}{l}\text { Sea surface temperature } \\
\text { (SST) }\end{array}$ & ECMWF \\
\hline $\begin{array}{l}\text { Surface wind variability } \\
\text { indicator }\end{array}$ & ASCAT \\
\hline
\end{tabular}

to improve the characterization of the GNSS-R observable. Finally, conclusions are presented in Section V.

\section{DATA AND Simulator}

\section{A. Data}

SGR-ReSI has operated in two different modes. From September 2014 to April 2015, it operated in unmonitored automatic gain control (UAGC) mode, in which the receiver absolute power levels are unknown since the instrument automatically adjusts the receiver gain to make optimal use of the available dynamic range. As from May 2015, it operates in fixed gain mode (FGC), which is more suitable for calibration purposes. In both cases, the DDMs are generated onboard at $1 \mathrm{~Hz}$ with a coherent integration time of $1 \mathrm{~ms}$. The entire SGR-ReSI L1B data set is collocated with ECMWF wind/wave parameters and ASCAT-A L2 winds. Different ECMWF/ASCAT parameters used in the analysis are listed in Table I. The SGR-ReSI data are converted from the L1A onboard processed DDMs into a standard multiframe tag image file format, and separated into tracks and referenced to files with synchronized metadata [16]. The collocated ECMWF parameters are acquired by interpolating three ECMWF 3-h forecast fields on a $0.5625^{\circ}$ latitude/longitude grid both spatially and temporally to the data acquisition location and time. ASCAT data are collocated with SGR-ReSI specular point acquisitions using the following criteria: a maximum separation of $20 \mathrm{~min}$ in time and $25 \mathrm{~km}$ in space.

The observable is a quantity derived from the DDM which depends on the underlying wind/wave conditions. In this paper, a normalized reflected signal (SNR) is the primary observable used to develop the GNSS-R GMF [17]. The noise $n_{\text {DDM }}$ is defined as the average of the noise floor across all Doppler bins and the first 20 negative delay bins of the DDM, as indicated by the red rectangle in Fig. 1. While, the signal is defined as the average of the peak power (denoted as $p_{\text {DDM }}$ ) across certain Doppler and delay bins (3 Doppler bins $\times 1$ delay bin, as indicated by the gray rectangle) subtracted by the noise. In practice, the peak position is directly determined from the DDM data. If the peak position is found at a Doppler shift lower than $-500 \mathrm{~Hz}$ or larger than $+500 \mathrm{~Hz}$, the corresponding DDM is excluded from the study. The raw SNR computation is based on [17], i.e.,

$$
\mathrm{SNR}_{0}=\frac{p_{\mathrm{DDM}}-n_{\mathrm{DDM}}}{n_{\mathrm{DDM}}} .
$$




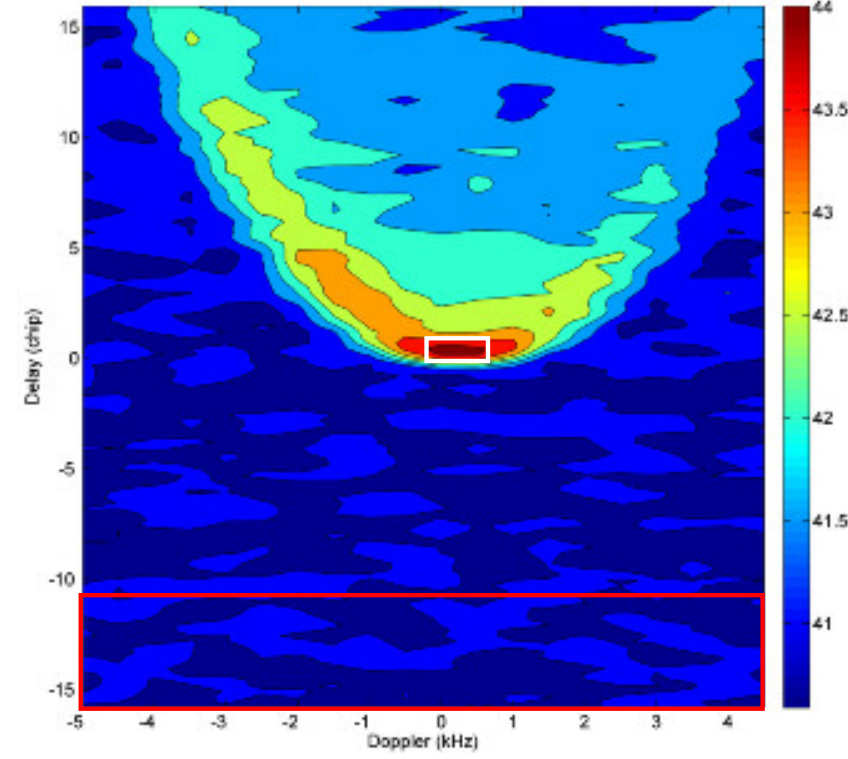

Fig. 1. Illustration of the observable definition: the noise is defined as the DDM average over the red rectangle, and the signal is define as the DDM average over the white rectangle minus the noise.

Although for FGC mode measurements, the SNR is not strictly needed for calibration purposes, for consistency, the SNR is computed for both UAGC and FGC modes, and in both the simulation and the analysis of real TDS-1 data. Note that ideally, i.e., from the simulation point of view, the observables signal, SNR, peak, and peak-to-noise ratio (PNR) are equivalent.

The SGR-ReSI DDMs do not always present the typical shape shown in Fig. 1, due to ice contamination or low SNR conditions. Therefore, a quality control (QC) together with the quality flags in the L1B product is needed to filter out poor-quality data before the analysis. First, all the measurements with SST $<-1{ }^{\circ} \mathrm{C}$ are excluded to avoid ice contamination. This is similar to the ice flagging used by the ASCAT L2 processing. Second, a four-sigma QC approach is used to filter out the noisy DDMs, i.e., the measurements with $\left(p_{\mathrm{DDM}} / \sigma_{n}\right)<4$ are excluded, where $\sigma_{n}$ is the standard deviation (SD) over the noise box. Finally, the DDMs whose peak positions are too far from the zero Doppler-zero delay bin (as given by the L1B data) are excluded. In practice, if the position of the maximum value is more than two Doppler bins or five delay bins away from the specular point, the corresponding DDM is excluded from the further analysis. The last two conditions usually correspond to measurements at high incidence angles and low $G_{r}$ values (less than $0 \mathrm{~dB}$ ), that is about $47 \%$ of the observations over the ocean.

\section{B. Simulator}

A GNSS-R open source end-to-end simulator, namely, Wavpy [17], is used to verify each step of the correction in this section. The simulator uses the observation geometries of the real data as input and generates DDMs at $1 \mathrm{~Hz}$ as well. Then the corrections are consolidated with real data analysis
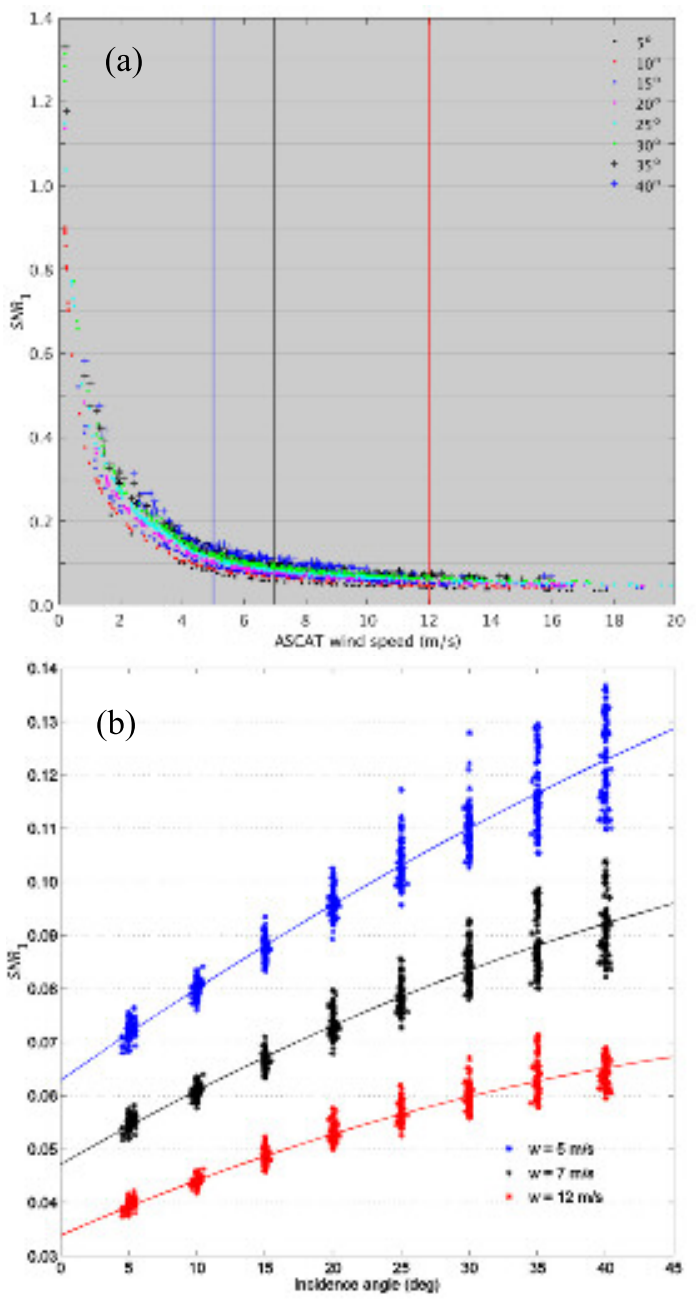

Fig. 2. (a) Simulated $\mathrm{SNR}_{1}$ as a function of wind speed ( $x$-axis) and incidence angle (color). (b) Same $\mathrm{SNR}_{1}$ (2) as a function of incidence and the fit regression cureve in solid, for three different wind speeds, i.e., 5, 7, and $12 \mathrm{~m} / \mathrm{s}$ [as shown by the vertical lines in (a)].

in Section III. The primary issue of the GMF development is to correct for the effect of receiver antenna gain, which dominates the observable variations. The correction is written as

$$
\mathrm{SNR}_{1}=\frac{\mathrm{SNR}_{0}}{G_{r}} .
$$

Fig. 2(a) shows the corrected $\mathrm{SNR}\left(\mathrm{SNR}_{1}\right)$, derived from a Wavpy simulation that uses the real TDS-1 settings available from the MERRByS product $\left(G_{r}\right.$, observation geometry, and $G_{t}$ ), as a function of ASCAT wind speed and for different incidence angle values (see legend). It is clear that $\mathrm{SNR}_{1}$ shows good correlation with the input wind speed for each incidence angle bin. However, this observable has a residual dependence on incidence angle. The main reason for this is that the following effects of the BRE have not been accounted for.

1) the surface area $(A)$ corresponding to the signal glistening area;

2) the transmitter power and its antenna gain $\left(G_{t}\right)$;

3) distance from the signal glistening area to the receiver/transmitter $\left(R_{r} / R_{t}\right)$. 
Ideally, the abovementioned effects should be corrected using the BRE approach as described in [11]. However, the actual implementation of BRE is time consuming, particularly for the calculation of surface area A. Moreover, different GPS satellites may have different transmitter power and antenna gain patterns, which need to be accounted for accurate $\sigma^{0}$ (and SNR) estimations. Since no accurate estimates of these parameters are available, we use an empirical approach to simplify the correction of the aforementioned effects. According to Fig. 2(b), the combined transmitted antenna power and gain (and residual receiver antenna gain) effect is a quasi-linear function of incidence angle, for all wind speeds. For simplicity, the combined effect on the SNR is simply modeled as a linear function of incidence angle $\theta$ in degrees

$$
f(\theta)=0.019426 \theta+0.93379 \text {. }
$$

The incidence angle correction $f(\theta)$ is then applied to $\mathrm{SNR}_{1}$ as

$$
\mathrm{SNR}_{2}=\frac{\mathrm{SNR}_{1}}{f(\theta)}
$$

The observable dependence on $f(\theta)$ is removed after applying (4) (not shown). However, small variations of $\mathrm{SNR}_{2}$ persist at certain incidence angle and wind speed bins. Such variations increase with $\theta[2 \%-6 \%$, see the small variations at each incidence bin in Fig. 2(b)]. This is because the sea surface area is indeed not only a function of $\theta$ but also slightly dependent on the observing azimuth (relative to the geographic north) and the relative motion between the receiver and the transmitter. If the surface area associated with the signal is estimated properly, such small variations can be totally removed [18].

\section{Methodology}

Following the simulation results, we apply the $G_{r}$ and $f(\theta)$ corrections to the real SGR-ReSI data. Then the 2-D histogram of the corrected $\mathrm{SNR}\left(\mathrm{SNR}_{2}\right)$ versus incidence angle $\theta$ is analyzed in order to verify the effectiveness of such corrections for real data. Note that in Fig. 3(a), the sampled wind probability density functions (PDFs) are dependent on incidence angle. Since the SGR-ReSI antenna is almost nadir looking, the capability of sensing the low signal power at high wind speeds decreases with increasing incidence angles, leading to a positive shift of the wind PDF for decreasing $\theta$. To discard any wind sampling effects in the residual dependence on $\theta$, a PDF matching technique is used. That is, the SGR-ReSI SNR 2 data are randomly selected to match the minimum number $N$ in each ECMWF speed bin as indicated by the black-bold curve in Fig. 3(a). Consequently, the 2-D histogram of $\mathrm{SNR}_{2}$ versus incidence angle $\theta$ is shown in Fig. 3(b). For the sake of comparison, each 2-D bin is normalized by the total number of samples within the corresponding incidence bin. The same approach is applied to the data acquired in FGC mode, which show similar results to those in Fig. 3.

Since the noise does not depend on $\theta$, the histogram of other observables, e.g., signal, peak, and PNR, is similar to that of the SNR ( $\mathrm{SNR}_{2}$, not shown). From Fig. 3(b), one can conclude that there is still incidence angle dependence for high incidence angles. This may be attributed to the Fresnel
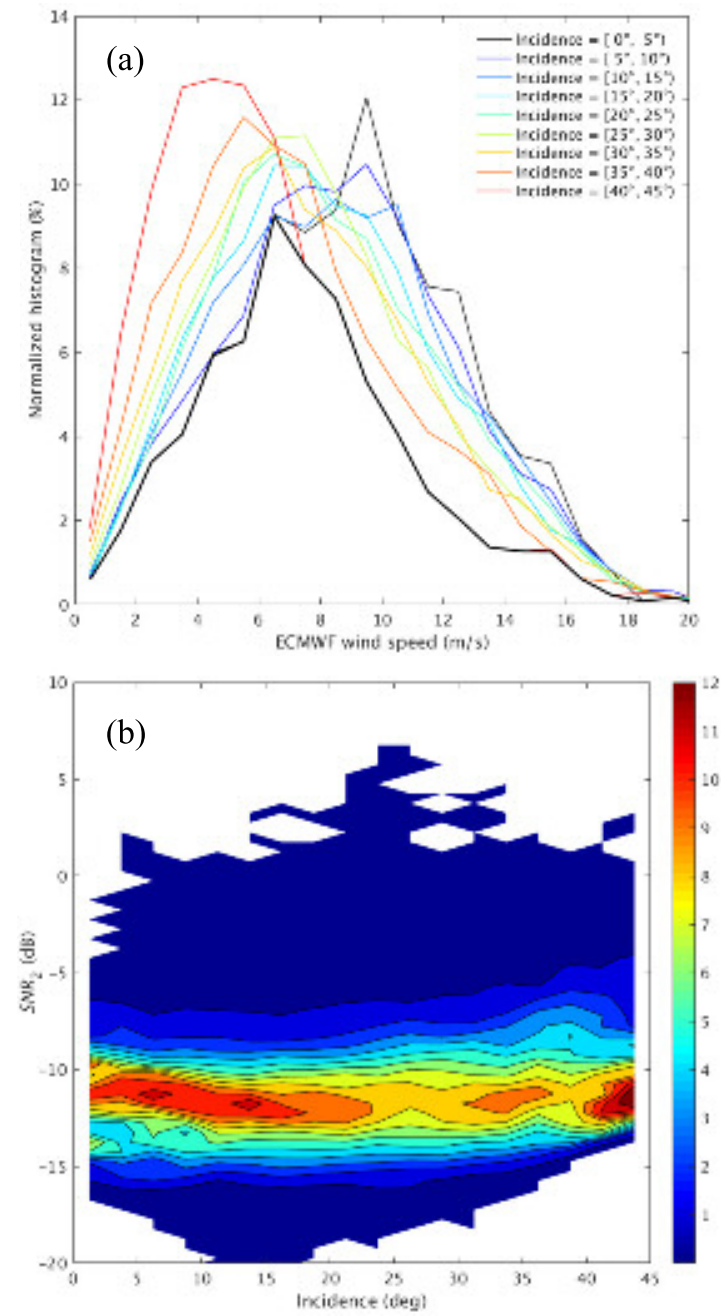

Fig. 3. (a) PDFs of wind speed for different incidence angle. (b) 2-D histogram of the real SGR-ReSI SNR 2 [i.e., after correcting for $G_{r}$ and $f(\theta)$ ] versus incidence angle. Data acquired at UAGC mode are used in these plots.

reflection coefficient [13], the uncalibrated receiver antenna gain and the unknown transmitter antenna gain. The Fresnel reflection coefficient is a function of incidence angle and dielectric permitivity. The latter is estimated using collocated ECMWF SST, a fixed sea surface salinity (SSS) value (35 psu), and the GPS L1 frequency (1575.42 MHz). The antenna gain patterns are not accounted for accurately in (2) and (3) and can be further considered as a function of both incidence angle and azimuth angle (i.e., the specular point to receiver vector in the receiver's antenna frame), as modeled by an additional calibration factor $\Delta \mathrm{g}(\theta, \varphi)$. In practice, on can estimate $\Delta \mathrm{g}$ using the following iterative process (see Fig. 4) and the following equation:

$$
\Delta g_{j, k}=\frac{1}{N} \sum_{i} \frac{n_{i} \cdot\left\langle x_{i, j, k}\right\rangle}{\left\langle x_{i}\right\rangle}
$$

where $x$ corresponds to the observable SNR, $N$ is the total amount of data, $n_{i}$ is the number of data in the $i$ th wind speed bin, and $j, k$ represents the bin number for incidence angle and azimuth angle, respectively. The bin size is $1 \mathrm{~m} / \mathrm{s}$ for $i, 1^{\circ}$ for $j$, and $10^{\circ}$ for $k$. 


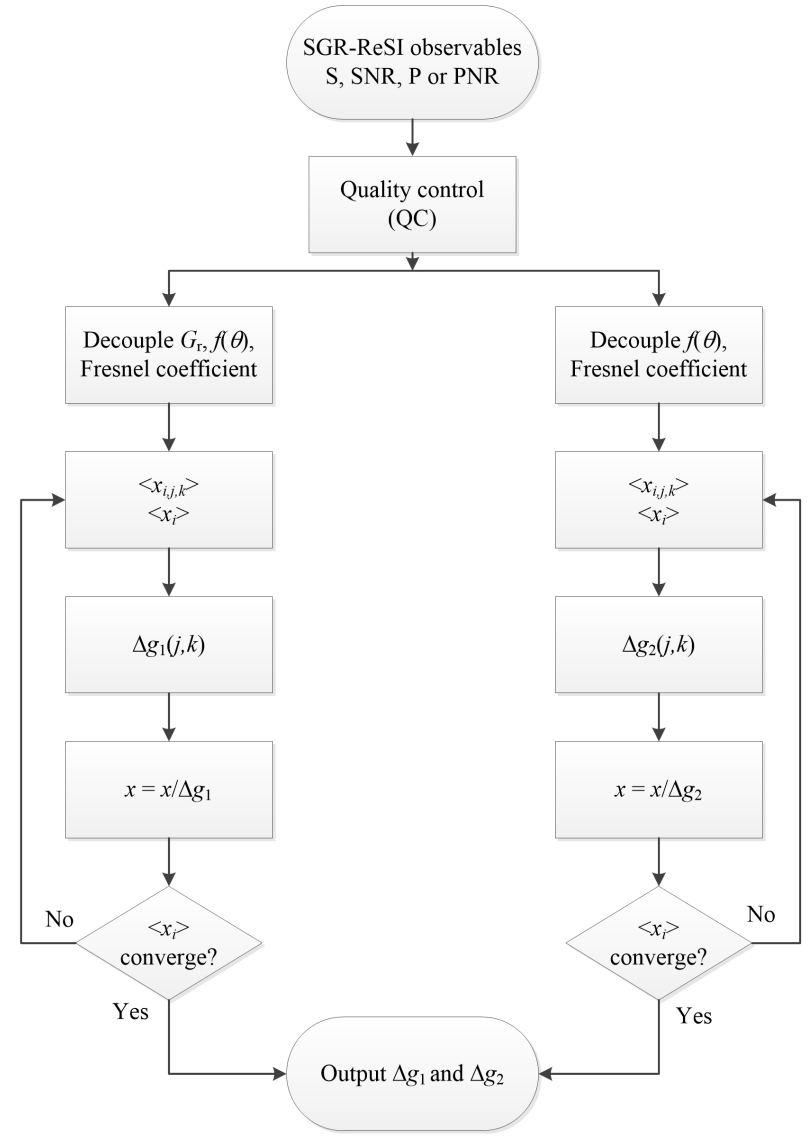

Fig. 4. Iterative process to estimate $\Delta \mathrm{g}$.

As shown in Fig. $4, \Delta \mathrm{g}_{1}$ corresponds to the contribution from the transmitter antenna gain and the unknown receiver antenna gain estimation (systematic) errors; while $\Delta \mathrm{g}_{2}$ corresponds to $\Delta \mathrm{g}_{1}$ and the estimated receiver antenna gain $G_{r}$ (given by the TDS-1 L1B product). The estimation of $\Delta \mathrm{g}_{2}$ is carried out to test the robustness of the iterative method. Indeed, by computing the ratio of $\Delta \mathrm{g}_{2}$ and $\Delta \mathrm{g}_{1}$, one should infer the receiver antenna gain pattern as

$$
G_{r}^{\text {inferred }}=\frac{\Delta g_{2}}{\Delta g_{1}}+C
$$

where $C$ is a constant value that makes $G_{r}^{\text {inferred }}$ and $G_{r}$ to have the same absolute value. It is independent of $\theta$ and $\varphi$ and does not affect the measurement scatters. Fig. 5 shows $\Delta \mathrm{g}_{1}$, $G_{r}^{\text {inferred }}$ and the estimated antenna gain pattern $G_{r}$ given by the SGR-ReSI L1B product, respectively. It is clear that the inferred and estimated receiver antenna patterns are almost identical (except for the high incidence angles), thus proving that the chart flow shown in Fig. 4 can be used to estimate the combined sytematic effects induced by the transmitter antenna gain and the residual receiver antenna gain errors.

In principle, SGR-ReSI receives signals from different GPS satellites, which may have different transmitter power and antenna gain pattern. Therefore, applying a single correction table $\Delta \mathrm{g}_{1}$ to all the transmitters may not lead to an optimal calibration. One should repeat the procedure shown in Fig. 4 for each transmitter separately, as specified

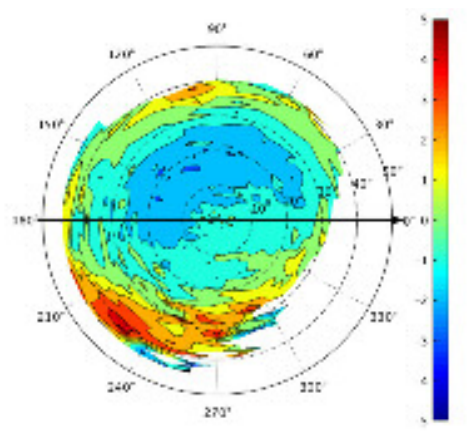

(b)

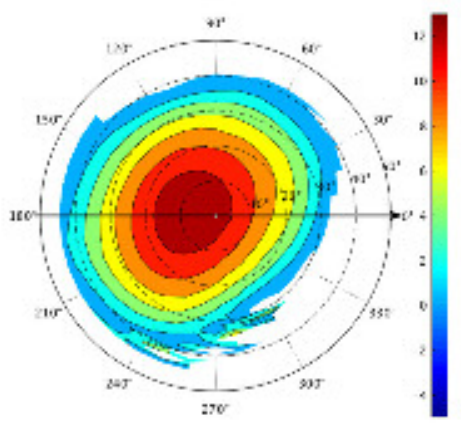

(c)

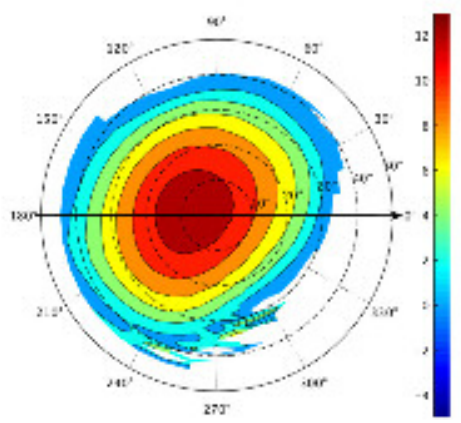

Fig. 5. (a) Additional calibration factor $\Delta g_{1}$ (combined receiver and transmitter $G$ ) as a function of elevation and azimuth angles. (b) Inferred receiver antenna gain pattern. (c) Estimated antenna gain pattern (given by the SGR-ReSI L1B product).

by the space vehicle numbers (SVNs), which can be easily transformed from the pseudorandom number (PRN) codes in the L1B product. Note that since very little amount of data are found from SVNs 26, 38, 70, and 73 during the study period (September 2014-April 2016), these transmitters are excluded from the analysis. After excluding these data sets, each PRN code corresponds to a unique SVN, such that one can use either SVN-dependent or PRN-dependent corrections in this paper. Finally, the observable of real data set is further corrected by the Fresnel reflection coefficient $\Re(\theta)$ [7] and the PRN-depdendent correction factor $\Delta g_{\text {PRN }}$

$$
\mathrm{SNR}_{3}=\frac{\mathrm{SNR}_{2}}{|\Re(\theta)|^{2} \cdot \Delta g_{\mathrm{PRN}}}
$$

Fig. 6 shows the same plot as Fig. 3(b) but for $\mathrm{SNR}_{3}$. It is clear that the incidence angle dependence is very much reduced by using the additional correction depicted by (7). Note though that the measurement uncertainties slightly increase 


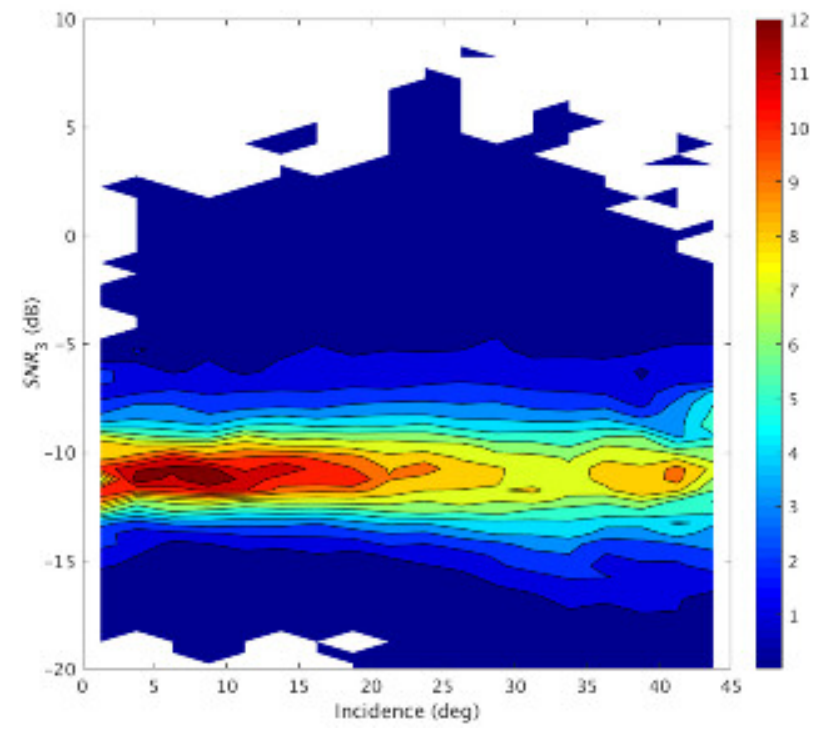

Fig. 6. 2-D histogram of the real SGR-ReSI $\mathrm{SNR}_{3}$ (after correcting for $G_{r}, f(\theta)$, Fresnel reflection coefficient and the PRN-dependent correction factor $\Delta \mathrm{g}_{\mathrm{PRN}}$ ) versus incidence angle for data in UAGC mode.

with incidence angle. After applying $\mathrm{QC}$ as described in Section II-A, all the valid observables $\mathrm{SNR}_{3}$ with $\theta<45^{\circ}$ and $G_{r}>0 \mathrm{~dB}$, i.e., $45 \%$ of the measurements over the ocean, are used in Section IV for the development of the wind GMF.

\section{RESULTS}

Following the definitions in Sections II and III, the observables $\mathrm{SNR}_{1}$ (2) and $\mathrm{SNR}_{2}$ (4) are further corrected by the Fresnel reflection coefficient $\Re(\theta)$ in order to ease the comparision between $\mathrm{SNR}_{1}$ (or $\mathrm{SNR}_{2}$ ) and $\mathrm{SNR}_{3}$. Hereafter, the subscripts below refer to the corrections to the observable SNR as follows:

1) subscript "1," observable after correcting for $G_{r}$, and Fresnel reflection coefficient;

2) subscript "2," observable after correcting for $G_{r}$, Fresnel reflection coefficient, and $f(\theta)$;

3) subscript " 3 ," observable after correcting for $G_{r}, f(\theta)$, Fresnel reflection coefficient, and $\Delta \mathrm{g}_{\text {PRN }}$.

Section IV-A presents the mean and the SD values of the studied observable as a function of wind speed. Then the observable characteristics are evaluated under different surface conditions according to the wind variability indicator included in the ASCAT data. Section IV-B addresses the impact of waves on the observable. The observable characteristics are evaluated for three different wave conditions, i.e., young sea, fully developed sea, and swell sea, as well as for different $H_{s}$ values.

\section{A. Observable Versus Winds}

1) SGR-ReSI-ECMWF Analysis: As shown in [18], the sensitivity of SNR to wind direction is negligible for all wind speed regimes. Therefore, the development of the wind GMF only takes the wind speed component into account. Fig. 7 shows the SD errors of different SNRs as a function of wind speed (FGC mode). It is clear that $\mathrm{SNR}_{3}$ has the

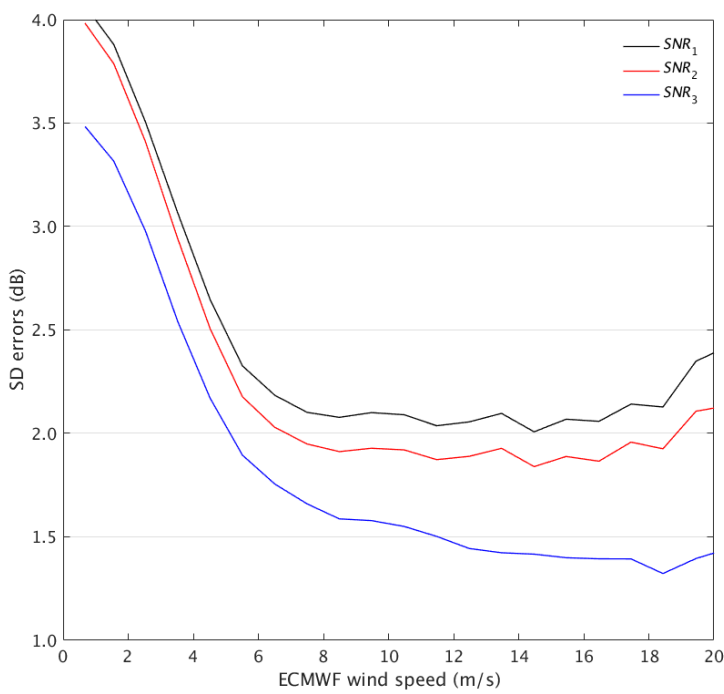

Fig. 7. SD errors as a function of wind speed for different SNRs (FGC mode).

lowest scatter, thus confirming that the corrections performed in Sections II and III are relevant for both forward modeling and inversion. Note though that different SNRs show a similar sensitivity to wind speed changes (not shown).

Fig. 8 shows the scatter-density plots of $\mathrm{SNR}_{3}$ versus ECMWF wind speed for different operation modes. The amount of UAGC and FGC data used is about 0.55 and 1.8 million, respectively. The magenta curve is the wind GMF derived from the Wavpy simulation. Note that a shift of $+1.5 \mathrm{~dB}$ has been applied to the Wavpy curve in order to match the real data highest density points. This bias is probably due to an overestimation of the noise, the instrument loss, or some other instrumental parameters in Wavpy. Since we are more interested on the relative changes (i.e., sensitivities) of the observables, this bias is not further addressed in this paper. Despite of the measurement scatter, the globally measured observable (SNR) has a very similar sensitivity as that of the simulated SNR from Wavpy. It also shows that the FGC mode has less uncertainty than the UAGC mode after applying the mentioned corrections. Moreover, the FGC mode shows larger sensitivity to high wind variations than the UAGC mode. Since the wind speed PDFs of UAGC and FGC are similar (not shown), only the data obtained in FGC mode are hereafter analyzed.

It is not so straightforward to compare SNR with the theoretical normalized bistatic normalized radar cross section (BNRCS), since the former contains the weighted combination of the scattering contribution from the specular point and the neighboring points, according to the BRE. Nevertheless, the measured SNR, in particular the $\mathrm{SNR}_{3}$, shows on average excellent agreement (within $0.3 \mathrm{~dB}$ for speeds between 2 and $14 \mathrm{~ms}^{-1}$ ) with the simulated BNRCS derived from Wavpy (not shown).

2) SGR-ReSI-ASCAT Analysis: Since the orbit of TDS-1 is quasi sun-synchronous, with a local time ascending node (LTAN) drift of $1.42 \mathrm{~h} / \mathrm{yr}$, the amount of collocated SGR-ReSI and ASCAT data is limited in FGC mode (TDS-1 LTAN was closest to that of Metop-ASCAT while 


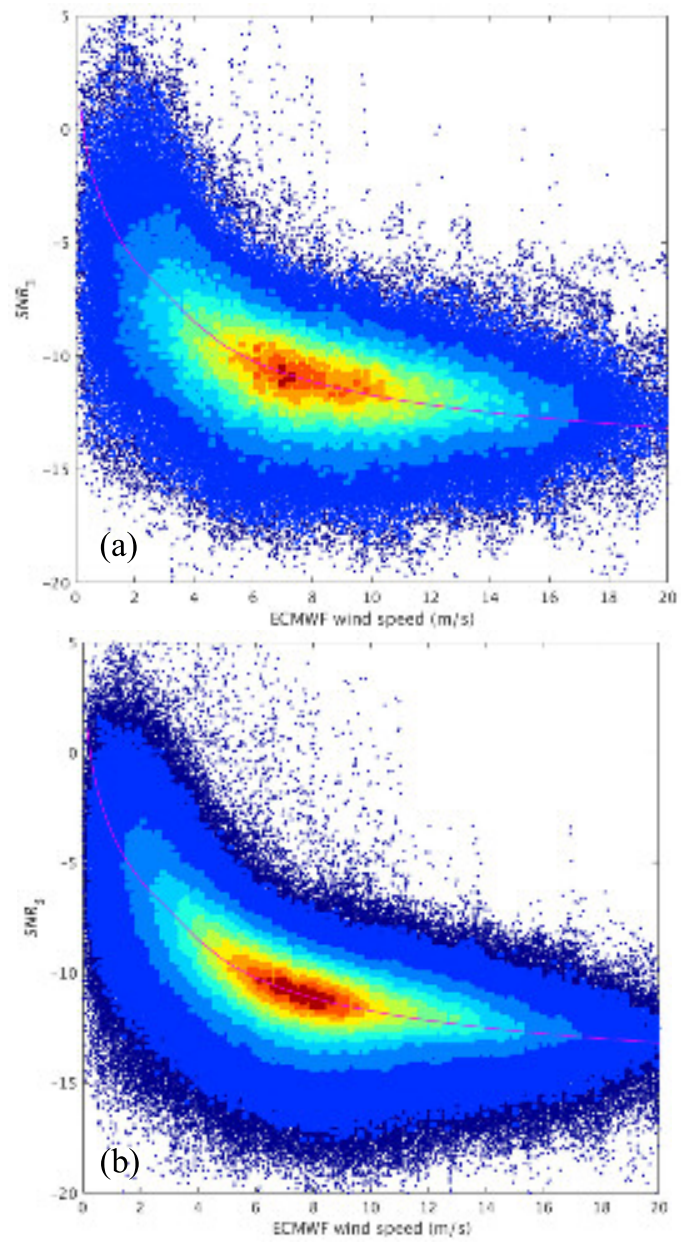

Fig. 8. Scatter-density plot of $\mathrm{SNR}_{3}$ versus ECMWF wind speed, for (a) UAGC mode and (b) FGC mode. The colors (from cool to warm) represent $1 \%, 10 \%, 20 \%, \ldots, 90 \%$ of the maximum number for the densest bin. The white curve shows the theoretical wind GMF derived from the Wavpy simulation

operating in UAGC mode, i.e., during the first six months of TDS-1 mission), and most of the collocations are located at mid and high latitudes. To avoid sampling issues, only triple collocated TDS-1/ASCAT/ECMWF data are used in this section. Fig. 9(a) shows that the $\mathrm{SNR}_{3}$ fit has less uncertainty and better fits the theoretical sensitivity (magenta curve) at low winds when using ASCAT winds (red curve) rather than ECMWF winds (black curve). This indicates that the SGR-ReSI actually measures the sea surface wind at a spatial resolution closer to that of ASCAT than that of ECMWF. Here, the error bars are purely empirical and can also be used as an indicator of the effective sensitivity of SNR to wind speed changes, i.e., the combination of the error bars and the sensitivity itself (as provided by the SNR slope as a function of wind speed). For example, a low wind sensitivity (e.g., at high winds) combined with a large scatter, leads to very large wind retrieval errors, which is equivalent to having a very low or marginal sensitivity with a small scatter.

The impact of wind variability on SGR-ReSI measurements is analyzed by simply checking the estimated SD errors of $\mathrm{SNR}_{3}$ for different ASCAT-derived wind variability conditions. As shown in [19], the singularity exponents (SEs)
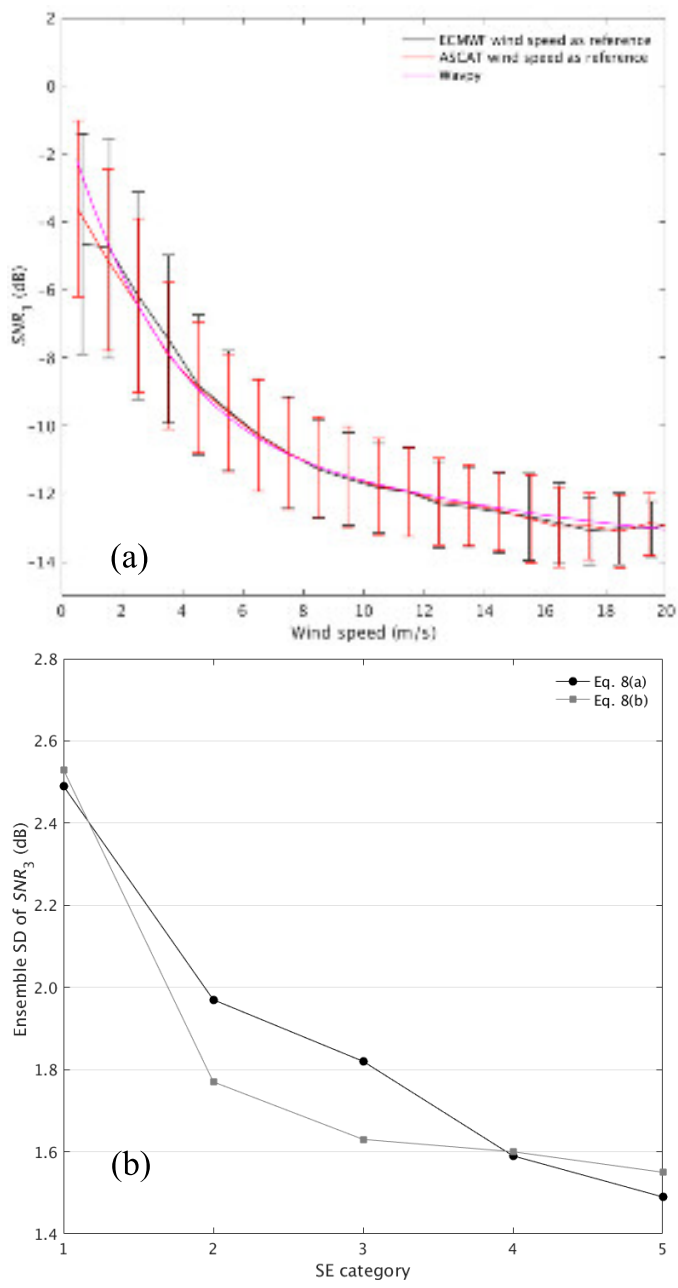

Fig. 9. (a) Mean $\mathrm{SNR}_{3}$ and corresponding $\mathrm{SD}$ errors (error bars) as a function of ECMWF wind speed (black curve), and ASCAT wind speed (red curve). The grey curve corresponds to the theoretical sensitivity derived from the Wavpy simulation. (b) Mean $\mathrm{SD}$ of $\mathrm{SNR}_{3}$ errors as a function of wind variability category.

derived from ASCAT data depict the degree of local regularity (spatial gradient) around a given point. The most negative singularity exponent (SE) values correspond to the least regular behavior of the ASCAT wind field (i.e., the highest wind variability conditions), while positive SE values indicate a more regular behavior (i.e., low wind variability conditions). In this section, a set of SE thresholds $(-0.15,-0.09,-0.05$, and 0 ) is used to roughly separate the surface wind variability into five different intervals or categories, such that the wind variability increases as the category index increases. In general, the slope of the mean $\mathrm{SNR}_{3}$ versus wind speed (i.e., the $\mathrm{SNR}_{3}$ sensitivity) does not change with wind variability (not shown). However, as seen in Fig. 9(b), the mean SD errors clearly increase with wind variability. Note that the mean SD values are derived from the following equations:

$$
\begin{aligned}
& \mathrm{SD}=\sqrt{\frac{\sum_{i=1}^{N_{s}} n_{i} \cdot \mathrm{SD}_{i}^{2}}{\sum_{i=1}^{N_{s}} n_{i}}} \\
& \mathrm{SD}=\sqrt{\frac{\sum_{i=1}^{N_{s}} 1 / n_{i} \cdot \mathrm{SD}_{i}^{2}}{\sum_{i=1}^{N_{s}} 1 / n_{i}}}
\end{aligned}
$$


where $i$ indicates the $i$ th wind speed bin (bin size $1 \mathrm{~m} / \mathrm{s}$ ), $n_{i}(5 \leq i \leq 12)$ is the percentile of samples in the $i$ th wind speed bin, and $\mathrm{SD}_{i}$ is the $\mathrm{SD}$ error for $\mathrm{SNR}_{3}$. For wind conditions of $w>12 \mathrm{~m} / \mathrm{s}$ or $w<5 \mathrm{~m} / \mathrm{s}$, there is too little data (e.g., only 10-300 samples) and, therefore, the results are not statistically significant. Equation 8 (a) is the common approach used to assess the ensemble mean value, while $8(\mathrm{~b})$ is usually adopted to mitigate the sampling effects (e.g., of wind speed). Taking the amount of data into account, the threesigma statistical uncertainties are about $0.17,0.16,0.14,0.10$, and $0.05 \mathrm{~dB}$ for the five SE categories, respectively. Such uncertainties are generally smaller than the difference between the corresponding adjacent categories (black curve). Therefore, these differences are statistically significant, except for the decrease of the mean SD values for the SE categories 4-5 when using 8(b) (gray curve). It can, therefore, be concluded that the $\mathrm{SNR}_{3} \mathrm{SD}$ errors generally increase as the wind variability increases (in particular, for categories 1-2).

\section{B. SNR Versus Nonwind Related Effects}

Here, we consider the sensitivity of SNR to wave parameters. As mentioned in Section I, the DDM is in theory fully characterized by MSS, which depends on the local wind speed, the fetch, and the incoming swell. Since wind is a significant contribution to MSS, these two variables are highly correlated [20] in the absence of swell. Therefore, one can either define an SNR-to-wind GMF like in Fig. 8 or an SNR-to-MSS GMF in a similar way (not shown). The question is what MSS data are available to develop an empirical SNR-to-MSS GMF? We first note that the high-frequency wave contribution to the MSS as sensed by GNSS reflectometry but not by the NWP model such as ECMWF. The ECMWF MSS (MSS WAM) represents the integral over all frequencies and directions of the 2-D wave spectrum (denoted as $\psi$ ) output from the ECMWF wave model (WAM), but with a high-frequency cutoff, whose wavenumber is $\kappa_{*}^{\mathrm{WAM}}=3.8 \mathrm{rad} / \mathrm{m}$ [21], while the TDS-1 MSS $\left(\mathrm{MSS}_{\mathrm{TDS}}\right)$ represents the same integral but with the following cutoff wavenumber [7], [22]:

$$
\kappa_{*}^{\mathrm{TDS}}=\frac{2 \pi \cos \theta}{3 \lambda}
$$

where $\theta$ is the incidence angle, and $\lambda$ is the wavenumber of the transmitted microwave signal. For TDS- $1, \kappa_{*}^{\mathrm{TDS}}=11 \mathrm{rad} / \mathrm{m}$ for $\theta=0^{\circ}$, and $\kappa_{*}^{\mathrm{TDS}}=7.8 \mathrm{rad} / \mathrm{m}$ for $\theta=45^{\circ}$. Therefore, the difference between MSSWAM and MSS TDS $_{\text {can }}$ be estimated as follows:

$$
\Delta \mathrm{mss}=\iint_{\kappa_{*}^{\mathrm{WAM}} \leq \kappa<\kappa_{*}} \kappa^{2} \psi(\kappa) d^{2} \kappa .
$$

Since $\kappa_{*}^{\text {TDS }}$ depends on the incidence angle, $\triangle$ MSS also shows dependence on the incidence angle, which in turn further complicates the development of an MSS GMF using ECMWF MSS as reference or the incorporation of MSS into the wind GMF. Moreover, the wave spectrum is a function of wind speed, indicating that the normalized difference between MSS $_{\text {TDS }}$ and MSS $_{\text {WAM }}$ (denoted as $\triangle$ MSS/MSS WAM $_{\text {) depends }}$ on wind speed as well (see Fig. 10). Here, the simulation

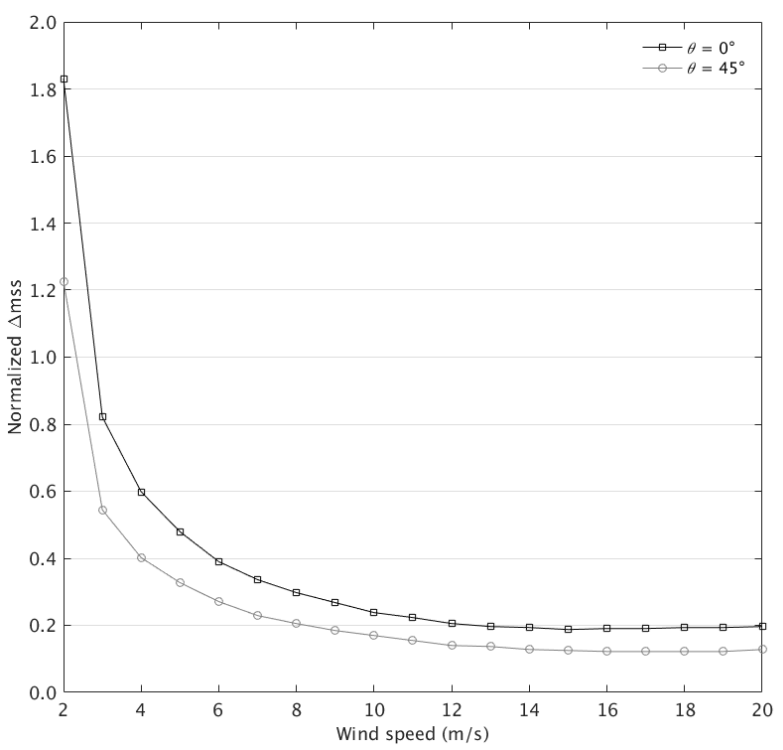

Fig. 10. Normalized $\mathrm{N} \Delta \mathrm{mss}\left(\Delta \mathrm{mss} / \mathrm{mss}_{\mathrm{WAM}}\right)$ as a function of wind speed for a fully developed sea state (Elfouhaly spectrum) and two different incidence angles (see legend)

is carried out for fully developed sea state using Elfouhaly spectrum [23]. It shows that $\triangle$ MSS decreases with increasing wind speed. Moreover, $\triangle \mathrm{MSS}$ is less prominent at higher incidence angle. Therefore, the development of a comprehensive MSS GMF and the analysis of residual MSS effects on the wind GMF are not considered at this stage.

1) Sea State: A qualitative approach is presented to assess the impact of long waves on SNR, i.e., the observable characteristics are evaluated under different sea-state conditions. A simple empirical categorization [24] is used to roughly discern between growing sea, fully developed sea and swell sea. This model includes three essential parameters, i.e., wind speed $U, H_{s}$, and an empirical threshold $t_{h}$ used to separate the different sea states. The significant wave height of fully developed sea is calculated from the Pierson-Moskowitz spectrum [25] and approximated by the following equation:

$$
H_{s}^{\mathrm{FD}} \cong 0.22 \frac{U^{2}}{g}
$$

where $g$ is the Earth's gravity acceleration. The normalized difference between $H_{s}^{\mathrm{FD}}$ and the estimated $H_{s}$ (from WAM) is computed as follows:

$$
d_{n}=\frac{H_{s}-H_{s}^{\mathrm{FD}}}{H_{s}^{\mathrm{FD}}} .
$$

Finally, the sea-state conditions are categorized as

$$
\begin{cases}d_{n}<-t_{h} & \text { growing sea } \\ d_{n}>t_{h} & \text { swell } \\ \text { otherwise } & \text { fully-developed sea }\end{cases}
$$

where the empirical threshold is set to $t_{h}=0.2$, such that the percentiles of growing sea, fully developed sea and swell sea are about 7\%, 23\%, and 70\%, respectively. Fig. 11 shows the mean $\mathrm{SNR}_{3}$ versus ASCAT wind speed for the different 


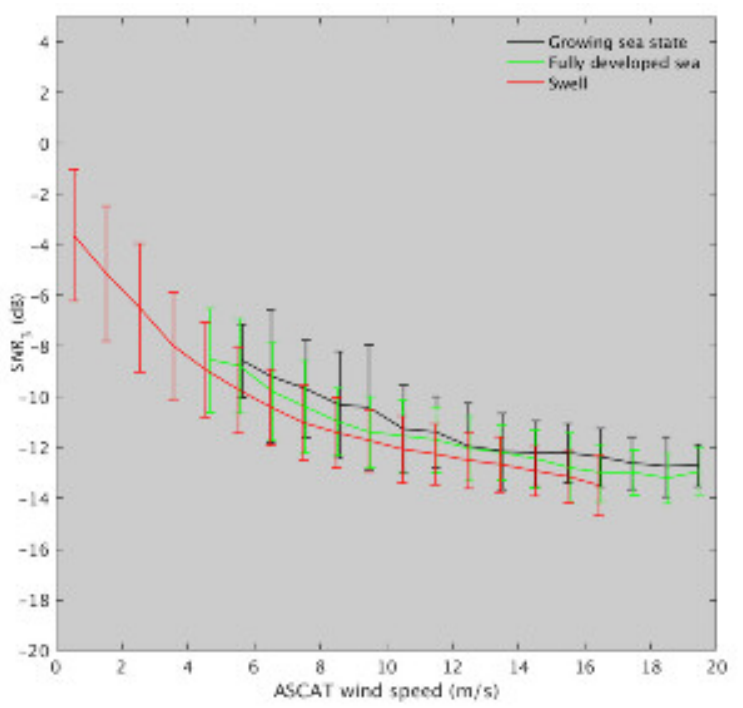

Fig. 11. Mean $\mathrm{SNR}_{3}$ and corresponding $\mathrm{SD}$ errors (error bars) as a function of ASCAT wind speed for different sea-state conditions.

sea-state conditions. Fully developed seas lead to lower SNR than younger seas. The impact of swell appears not negligible. Besides the discernible biases among the three sea-state categories, the observable shows larger uncertainties for growing sea state than for the other sea-state conditions. However, the SNR sensitivity to wind speed changes is rather similar for all conditions.

2) Significant Wave Height: Following (10), MSS is mostly determined by large wave numbers, hence short (wind) waves, while $H_{s}$ features larger sensitivity to swell waves. As such, ECMWF winds are more correlated with ECMWF MSS than to $H_{S}$ [20]. It is, therefore, worth analyzing whether the SNR dependence on $H_{S}$ is complementary to that on wind speed. Fig. 12 shows the mean $\mathrm{SNR}_{3}$ as a function of (a) ASCAT and (b) ECMWF wind speeds under different $H_{S}$ conditions. As expected, the observable is more sensitive to ASCAT winds than to ECMWF winds, since the SNR footprint is closer to ASCAT wind resolution than to ECMWF wind resolution. However, the sensitivity is very low for both wind references when $H_{S}>5 \mathrm{~m}$, since large $H_{S}$ values typically correspond to winds higher than $7 \mathrm{~m} / \mathrm{s}$. Fig. 12(c) shows the same as Fig. 12(b) but for the entire SGR-ReSI data set (i.e., not limited to the collocated SGR-ReSI-ASCAT data set). Note that in the former, the curves span over different number of wind speed bins than in the latter. The reason is obvious: in relative terms, the entire SGR-ReSI data set contains more median wave state conditions $\left(2 \mathrm{~m}<H_{s}<4 \mathrm{~m}\right)$ and less calm $\left(H_{S}<2 \mathrm{~m}\right) /$ extreme $\left(H_{S}>4 \mathrm{~m}\right)$ wave state conditions than the more limited ASCAT-collocated data set. Consequently, the blue and magenta curves in Fig. 12(c) are more likely to reach the global median wind speeds [see Fig. 12(b) and (c)]. Note that for winds below $7 \mathrm{~m} / \mathrm{s}$, the SNR sensitivity to wind speed changes with $H_{s}$, i.e., it increases with decreasing $H_{s}$. Consequently, adding an $H_{s}$ term in the wind GMF may be relevant. Although this may further complicate the wind inversion (i.e., one observable for two unknowns), Clarizia and Ruf [14] have shown that with a Bayesian approach, conditioned by prior information, the combined GMF leads to improved wind retrievals, particularly for low winds.

\section{Discussion}

The approach for the derivation of the GNSS-R observable in Sections II and III accounts for the receiver antenna gain effects, and models the combined effects of GNSS-R geometry, transmitter antenna gain, and surface area around the specular point in an empirical way. It further addresses the effect of uncalibrated antenna gain using an iterative method for each GPS satellite, leading to considerable reduction of uncertainty in the observables. In particular, the proposed empirical approach can be easily implemented using predefined regression coefficients (3) and correction LUT ( $\left.\Delta g_{\text {PRN }}\right)$, which is highly desired for fast-delivery ground processing. Future efforts are needed to refine the observable in order to improve the GNSS-R GMF. First, to quantify the surface area of the glistening zone accurately, which may further reduce the observable uncertainty by $2 \%-6 \%$. Second, to take SSS into account for the calculation of the Fresnel reflection coefficient $\Re(\theta)$, since cold water (polar regions) and rainy (tropics) areas usually correspond to significantly lower SSS value than eslewhere. Third, to characterize the noise effects. The estimated noise in Fig. 1 actually includes the instrument and antenna noise, as well as the thermal noise of atmosphere and earth surface (and, occasionally, reflections of radiation from the sun, the moon, and the milky way) intercepted by the antenna. It may be relevant as well to carry out the noise monitoring.

Physically speaking, MSS may be closer to the GNSS-R observable, which is dominated by reflection and scattering of waves on the local ocean topography. The reflection part is expected to depend on swell traveling within the footprint, but generated external to it. The high-frequency wave contribution is defined as wind sea and is indeed highly correlated with wind. If accurate MSS reference data were available and if GNSS-R indeed responded to MSS, then a MSS GMF would be obviously better than a wind GMF, since it accounts for the swell effect on MSS and wind does not. However, if no accurate MSS reference data are available, then a wind GMF may be preferred, since MSS and wind are highly correlated. Moreover, ASCAT winds are very good and have verified ECMWF model winds too, but MSS is relatively uncertain. It is essentially modeled from the winds and integrated in time. This is why a wind GMF is attractive and an MSS GMF is not.

The significant wave height is composed of wind sea and swell contributions. Although it is not an ideal variable to represent the nonlocal sea state, one may expect that $H_{S}$ is complementary to the analysis of SNR dependence on wind speed, particularly for high $H_{S}$ and low wind cases. However, since $H_{S}$ depends on wind speed $\left(U^{2}\right)$ too, separating the SNR into different $H_{s}$ categories generally suffers from conditional sampling errors drawn from different wind and error PDFs. The only way to get useful information out of underdetermined observations is to add prior knowledge. Ideally this should be fit in the context of a 4-D variational approach, though most 

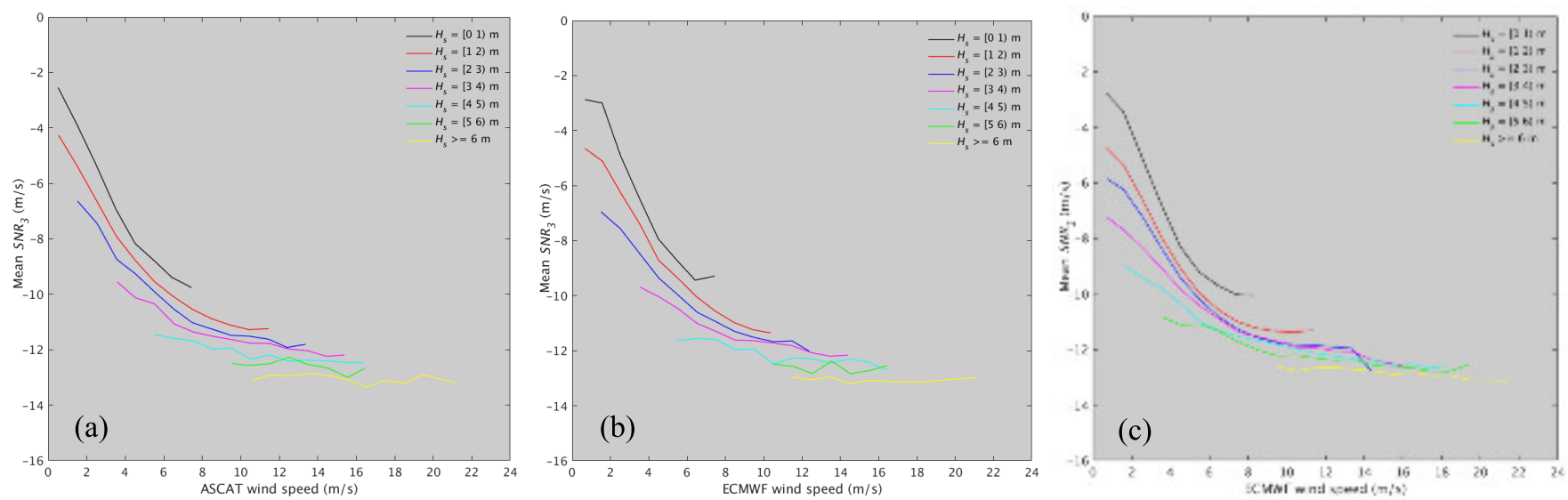

Fig. 12. Mean $\mathrm{SNR}_{3}$ as a function of (a) ASCAT and (b) ECMWF wind speeds for the collocated SGR-ReSI and ASCAT data set. (c) Same as (b) but for the entire SGR-ReSI data set (i.e., not limited to the collocated SGR-ReSI-ASCAT data set).

NWP and WAMs are not integrated unfortunately. In that case, one may use the prior $H_{s}$ from ECMWF to retrieve a better wind speed. $H_{S}$ errors in the prior are then projected on the GNSS-R speed error, so this should be used with caution.

\section{CONCLUSION}

In this paper, both the simulation and the analysis of the SGR-ReSI L1B data are used to develop the GNSS-R GMFs. The simulation results show that an SNR correction for the receiver antenna gain $G_{r}$ and the incidence angle $f(\theta)$ is essential for forward model development purposes. Actually, $f(\theta)$ includes the effects of the receiver and transmitter geometry and the specular area. Consequently, an innovative method is developed to further correct for the residual incidence dependence in the real data, which may be attributed to the uncalibrated receiver antenna gain and the unknown transmitter antenna gain, and is modeled as a function of both incidence angle and azimuth angle. In practice, such residual incidence dependence is modeled as a 2-D LUT. To account for different $G_{t}$ and transmitter power $\left(P_{t}\right)$ characteristics, the LUT corrections are applied for each satellite ID or PRN. The overall corrections remarkably reduce the measurement uncertainties, and as such, are the basis for the GNSS-R GMF development.

The results show that ASCAT winds are more representative of TDS-1 SNRs than ECMWF winds, and, therefore, more suitable for defining the GMF, particularly for wind speeds below $3 \mathrm{~m} / \mathrm{s}$. SNRs observed in the fixed gain control (FGC) mode are more sensitive to surface wind than those observed in the UAGC mode. An empirical wind-only GMF is then generated, which sensitivity agrees well with the physically based model used in the simulation procedure.

The SNR dependence on SST is not investigated. At low SST conditions, the large SD values of the SGR-ReSI measurements are caused by the high wind variability conditions at high latitudes. Although the observable uncertainties do depend on wind variability, no significant dependence for the noise estimates is found. Therefore, no error model can be developed at this stage for GNSS-R wind inversion purposes.

Sea surface winds and MSS are highly correlated, thus one can either use a wind GMF or an MSS GMF.
However, due to the lack of reliable MSS reference, no MSS GMF development or MSS impact analysis on the wind GMF has been carried out. It is recommended to further assess the SNR-to-MSS relationship with a reference MSS that accounts for the different wavelength cutoffs between GNSS-R and model output. A comprehensive Wavpy simulation can be carried out to compute such $\Delta$ MSS, which can be used to correct the auxiliary (model) MSS estimates. In line with [14], a nonnegligible dependence of SNR on significant wave height is found, complementary to that on wind. In particular, the SNR sensitivity increases for decreasing $H_{S}$ values, at winds below $7 \mathrm{~m} / \mathrm{s}$.

\section{ACKNOWLEDGMENT}

The authors would like to thank the two reviewers who helped to improve the paper.

\section{REFERENCES}

[1] V. Zavorotny et al., "Seasonal polarimetric measurements of soil moisture using tower-based GPS bistatic radar," in Proc. IEEE Int. Geosci. Remote Sens. Symp. (IGARSS), vol. 2. Piscataway, NJ, USA: IEEE Press, Jul. 2003, pp. 781-783.

[2] D. Masters, P. Axelrad, and S. Katzberg, "Initial results of land-reflected GPS bistatic radar measurements in SMEX02," Remote Sens. Environ., vol. 92, no. 4, pp. 507-520, Sep. 2004.

[3] S. J. Katzberg, O. Torres, M. S. Grant, and D. Masters, "Utilizing calibrated GPS reflected signals to estimate soil reflectivity and dielectric constant: Results from SMEX02," Remote Sens. Environ., vol. 100, no. 1, pp. 17-28, Jan. 2006.

[4] M. Wiehl, B. Legrésy, and R. Dietrich, "Potential of reflected GNSS signals for ice sheet remote sensing," Prog. Electromagn. Res., vol. 40, pp. 177-205, 2003.

[5] A. Komjathy, J. Maslanik, V. U. Zavorotny, P. Axelrad, and S. J. Katzberg, "Sea ice remote sensing using surface reflected GPS signals," in Proc. IEEE Int. Geosci. Remote Sens. Symp. (IGARSS). Piscataway, NJ, USA: IEEE Press, Jul. 2000, pp. 2855-2857.

[6] V. U. Zavorotny and C. Zuffada, "A novel technique for characterizing the thickness of first-year sea ice with the GPS reflected signal," in Proc. Eos Trans. AGU, Fall Meet. Suppl., 2002, vol. 83, no. 47, Paper C11A-0980.

[7] V. U. Zavorotny and A. G. Voronovich, "Scattering of GPS signals from the ocean with wind remote sensing application," IEEE Trans. Geosci. Remote Sens., vol. 38, no. 2, pp. 951-964, Mar. 2000.

[8] E. Cardellach, G. Ruffini, D. Pino, A. Rius, A. Komjathy, and J. L. Garrison, "Mediterranean Balloon Experiment: Ocean wind speed sensing from the stratosphere, using GPS reflections," Remote Sens. Environ., vol. 88, no. 3, pp. 351-362, 2003. 
[9] A. Komjathy, M. Armatys, D. Masters, P. Axelrad, V. Zavorotny, and S. Katzberg, "Retrieval of ocean surface wind speed and wind direction using reflected GPS signals," J. Atmos. Ocean. Technol., vol. 21, no. 3, pp. 515-526, Mar. 2004.

[10] M. Unwin, P. Jales, J. Tye, C. Gommenginger, G. Foti, and J. Rosello, "Spaceborne GNSS-reflectometry on TechDemoSat-1: Early mission operations and exploitation," IEEE J. Sel. Topics Appl. Earth Observ. Remote Sens., vol. 9, no. 10, pp. 4525-4539, Oct. 2016.

[11] G. Foti et al., "Spaceborne GNSS reflectometry for ocean winds: First results from the UK TechDemoSat-1 mission," Geophys. Res. Lett., vol. 42, no. 13, pp. 5435-5441, Jul. 2015.

[12] M. P. Clarizia, C. S. Ruf, P. Jales, and C. Gommenginger, "Spaceborne GNSS-R minimum variance wind speed estimator," IEEE Trans. Geosci. Remote Sens., vol. 52, no. 11, pp. 6829-6843, Nov. 2014.

[13] M. Clarizia and C. S. Ruf, "Wind speed retrieval algorithm for the Cyclone Global Navigation Satellite System (CYGNSS) mission," IEEE Trans. Geosci. Remote Sens., vol. 54, no. 8, pp. 4419-4432, Aug. 2016.

[14] M. P. Clarizia and C. S. Ruf, "Bayesian wind speed estimation conditioned on significant wave height for GNSS-R ocean observations," J. Atmos. Ocean. Technol., vol. 34, pp. 1193-1202, May 2017.

[15] Z. Wang et al., "SST dependence of $\mathrm{Ku}$ - and C-band backscatter measurements," IEEE J. Sel. Topics Appl. Earth Observ. Remote Sens., vol. 10, no. 5, pp. 2135-2146, May 2017.

[16] P. Jales, "MERRByS product manual-GNSS Reflectometry on TDS-1 with the SGR-ReSI," Surrey Satellite Technol. Ltd., Guildford, U.K., Tech. Rep. 0248366, May 2016.

[17] S. Soisuvarn, Z. Jelenak, F. Said, P. Chang, and A. Egido, "The GNSS reflectometry response to the ocean surface winds and waves," IEEE J. Sel. Topics Appl. Earth Observ. Remote Sens., vol. 9, no. 10, pp. 4678-4699, Oct. 2016.

[18] F. Fabra, E. Cardellach, W. Li, and A. Rius, "WAVPY: A GNSS-R open source software library for data analysis and simulation," in Proc. IEEE Int. Geosci. Remote Sens. Symp. (IGARSS), Fort Worth, TX, USA, Jul. 2017, pp. 4125-4128.

[19] W. Lin, M. Portabella, A. Stoffelen, J. Vogelzang, and A. Verhoef, "ASCAT wind quality under high subcell wind variability conditions," J. Geophys. Res. Oceans, vol. 120, no. 8, pp. 5804-5819, Aug. 2015.

[20] W. Lin and M. Portabella, "TGSCATT technical note 3: Generation of geophysical model functions for GNSS-R," Inst. Marine Sci., Spanish Nat. Res. Council, Barcelona, Spain, Tech. Rep. ESA:AO 18365/15/NL/CT, 2017.

[21] P. Janssen, J.-R. Bidlot, S. Abdalla, and H. Hersbach, "Progress in ocean wave forecasting at ECMWF," in Proc. ECMWF Tech. Memorandum, 2005, pp. 9-13.

[22] G. R. Valenzuela, "Theories for the interaction of electromagnetic and oceanic waves-A review," Boundary-Layer Meteorol., vol. 31, nos. 1-4, pp. 61-85, 1978.

[23] T. Elfouhaily, B. Chapron, K. Katsaros, and D. Vandemark, "A unified directional spectrum for long and short wind-driven waves," J. Geophys. Res., vol. 102, no. C7, pp. 15781-15796, 1997.

[24] G. Grieco, W. Lin, M. Migliaccio, F. Nirchio, and M. Portabella, "Dependency of the Sentinel-1 azimuth wavelength cut-off on significant wave height and wind speed," Int. J. Remote Sens., vol. 37, no. 21, pp. 5086-5104, 2016.

[25] W. Pierson, Jr., and L. Moskowitz, "A proposed spectral form for fully developed wind seas based on the similarity theory of S. A. Kitaigorodskii," J. Geophys. Res., vol. 69, no. 24, pp. 5181-5190, 1964.

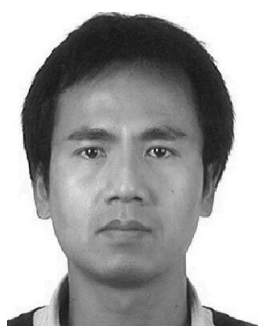

Wenming Lin (M'16-SM'16) was born in Fujian, China, in 1984. He received the B.Sc. degree in engineering from Wuhan University, Wuhan, China, in 2006, and the Ph.D. degree in engineering from the National Space Science Center, Chinese Academy of Sciences, in 2011.

He was a Post-Doctoral Researcher with the Institut de Ciències del Mar, Barcelona, Spain, where he is involved in advanced oceanographic data processing methods, remote sensing of ocean-surface winds, and data assimilation from 2011 to 2017 . He is currently with the Nanjing University of Information Science and Technology, Nanjing, China.

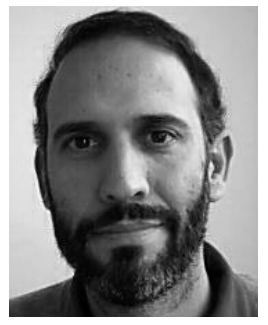

Marcos Portabella was born in Barcelona, Spain, 1970. He received the B.Sc. degree in physics from the University of Barcelona, Barcelona, in 1994, the M.Sc. degree in remote sensing from the Institute of Space Studies of Catalonia, Barcelona, in 1995, and the $\mathrm{Ph} . \mathrm{D}$. degree in physics, from the University of Barcelona, in 2002

$\mathrm{He}$ is currently with the Institut de Ciències del Mar, Barcelona, Spain, where he is involved in satellite remote sensing, particularly in scatterometry and L-band radiometry.

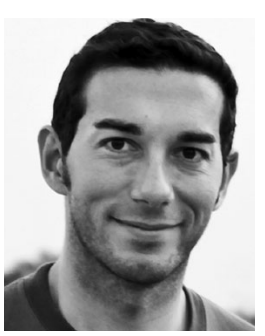

Giuseppe Foti received the M.Eng. degree in electronics engineering from the University of Catania, Catania, Italy, in 2000, and the M.Sc. degree in oceanography from the University of Southampton, Southampton, U.K., in 2013.

In 2001, he joined the Communication Systems Section, European Space Agency, Noordwijk, The Netherlands, where he was involved in the field of spread-spectrum techniques for packet access in broadband satellite systems. From 2003 to 2010, he was a Patent Examiner with the Principal Directorate of Telecommunications, European Patent Office, Rijswijk, The Netherlands. In 2013, he joined the Satellite Oceanography Section, National Oceanography Centre, Southampton, U.K., where he is currently a Research Scientist. His research interests include remote sensing of the oceans, with special emphasis on techniques using signals of opportunity.

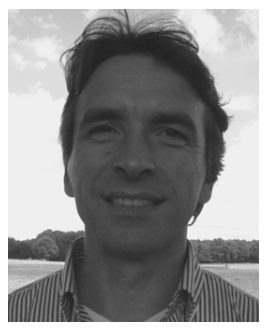

Ad Stoffelen (M'13-SM'14) received the M.Sc. degree in physics from the Technical University of Eindhoven, Eindhoven, The Netherlands, in 1987, and the Ph.D degree in meteorology on scatterometry from the University of Utrecht, Utrecht, The Netherlands, in 1998.

$\mathrm{He}$ is currently leading a group on active satellite sensing with Royal Netherlands Meteorological Institute, De Bilt, The Netherlands. His research interests include future missions and research and development for retrieval to $24 / 7$ operations, NWP mesoscale wind data assimilation, user training, and services, establishing an international scatterometer virtual constellation, and ESA ADM-Aeolus Doppler Wind Lidar mission.

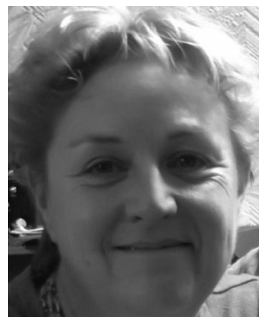

Christine Gommenginger received the Diplome d'Etudes Approfondies degree in electromagnetics, telecommunications, and remote sensing from the University of Toulon, Toulon, France, and the University of Nice Sophia Antipolis, Nice, France, and the Ph.D. degree in microwave radar remote sensing of the ocean at low grazing angles from the University of Southampton, Southampton, U.K.

She was with the National Oceanography Centre, Southampton, for more than 20 years. Her research interests include active and passive microwave remote sensing of the ocean, understanding interactions of microwave signals with the ocean surface, remote sensing of ocean wind and waves and developing new earth observation technologies and applications, altimetry for sea state, along-track interferometric synthetic aperture radar (SAR) for currents, global navigation satellite system reflectometry for surface winds and sea state, SAR altimetry, salinity from space with soil moisture ocean salinity, and wide-swath ocean altimetry. 


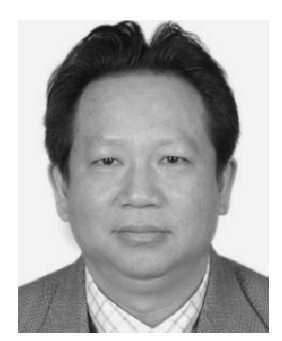

Yijun He (M'03) received the B.S. degree in physics from Hunan Normal University, Changsha, China, in 1985, the M.S. degree in applied physics from Xidian University, Xi'an, China, in 1990, and the $\mathrm{Ph} . \mathrm{D}$. degree in microwave theory and technology from Southeast University, Nanjing, China, in 1993.

$\mathrm{He}$ is currently a Professor of ocean remote sensing and the Dean of the School of Marine Sciences, Nanjing University of Information Science and Technology, Nanjing, China. He was with the Key Laboratory of Ocean Circulation and Waves, Institute of Oceanology, Chinese Academy of Sciences, Qingdao, China, where he was the Head of the Remote Sensing Group and Senior Scientist in Satellite Oceanography from 1996 to 2011. He was a Post-Doctoral Fellow with the Ocean Remote Sensing Institute, Ocean University of China, Qingdao, from 1993 to 1996 . He was a Visiting Scientist with the University of Hamburg, Hamburg, Germany, the Bedford Institute of Oceanography, Dartmouth, NS, Canada, and the University of Delaware, Newark, DE, USA. His research interests include ocean waves, sea-surface wind speed, other ocean-surface features related to remote sensing by full polarization synthetic aperture radar and other microwave radar at low incidence angle, air-sea gas exchange using microwave remote sensing and numerical models, sea-surface scattering of electromagnetic waves and mesoscale, and submesoscale ocean dynamics. 